Bond University

Research Repository

\title{
Calorie-Containing Recovery Drinks Increase Recreational Runners' Voluntary Energy and Carbohydrate Intake, with Minimal Impact on Fluid Recovery
}

Ben Desbrow; Barnes, Katelyn; Cox, Gregory R.; ludakhina, Elizaveta; McCartney, Danielle; Skepper, Sierra; Young, Caroline; Irwin, Chris

Published in:

International Journal of Sport Nutrition and Exercise Metabolism

DOI:

10.1123/ijsnem.2019-0043

Licence:

Other

Link to output in Bond University research repository.

Recommended citation(APA):

Ben Desbrow, Barnes, K., Cox, G. R., Iudakhina, E., McCartney, D., Skepper, S., Young, C., \& Irwin, C. (2019).

Calorie-Containing Recovery Drinks Increase Recreational Runners' Voluntary Energy and Carbohydrate Intake, with Minimal Impact on Fluid Recovery. International Journal of Sport Nutrition and Exercise Metabolism, 29(4), 359-363. https://doi.org/10.1123/ijsnem.2019-0043

\section{General rights}

Copyright and moral rights for the publications made accessible in the public portal are retained by the authors and/or other copyright owners and it is a condition of accessing publications that users recognise and abide by the legal requirements associated with these rights.

For more information, or if you believe that this document breaches copyright, please contact the Bond University research repository coordinator. 
2 Post-exercise "recovery stations" (where food and fluid are available) are a common feature of

3 developed sports programs for athletes and routinely organised for mass participation events

4 (e.g. fun runs, marathons, triathlons). While these are often critical to promote recovery for

5 individuals training or competing multiple times a day, this is less so for recreational athletes,

6 as both fluid and substrate losses can be restored within 24hrs (Burke, van Loon, \& Hawley,

7 2017; B. Desbrow, Barnes, Young, Cox, \& Irwin, 2017). Indeed, the consumption of caloric

8 beverages in the immediate post-exercise period could result in undesirable outcomes. For

9 instance, we have recently demonstrated that ad libitum access to calorie containing beverages

(i.e. carbohydrate (CHO)-electrolyte (sports) beverages and milk-based drinks) in the

laboratory, increases acute energy intake (in both males and females)(Campagnolo et al., 2017;

McCartney, Irwin, Cox, \& Desbrow, 2018). Hence, the immediate provision of food and calorie-containing beverages to recreational athletes could assist in meeting post-exercise recovery nutrition goals or alternatively, compromise broader health or body composition goals; particularly if subsequent dietary intake is not adjusted to compensate for the additional post-exercise intake.

To date, only one study has examined if the provision of foods/fluids at a recovery station immediately following a mass-participation event influences post-exercise dietary intake. In this study, providing ad libitum access to water, a commercial sports drink and sliced fruit in a recovery area had a positive influence on dietary intake (increasing fruit consumption) compared to when no recovery area was available (B. Desbrow et al., 2017). However, access to a recovery station did not influence total fluid or macronutrient intake across the remainder of the day or next morning hydration status (Urine Specific Gravity $\left(\mathrm{U}_{\mathrm{SG}}\right)$ ). Hence, it was concluded that recovery stations served to promote positive lifestyle behaviors in recreational athletes. 
Food/fluid items within recovery stations are not standardized, and providing/restricting access

to certain foods/fluids is likely to influence recovery and subsequent intake. In the former investigation, a range of items were provided including multiple beverages (calorie and caloriefree options) as well as sliced fruit (B. Desbrow et al., 2017), which are likely to have affected the recovery area's potential to influence subsequent dietary intake. In contrast, many events have limited resources (i.e. funds, personnel, capacity to handle perishable items), which dictate that access to fluid is typically prioritized. Hence, understanding how (if at all) access to different beverages (alone) immediately following recreational exercise influences dietary intake will assist in the development of recommendations for recovery provisions following mass participation events where limited resources exist.

In laboratory settings, the exclusive provision of commercial sports drinks (vs water), appears to result in an increase to ad libitum fluid intakes, both during (Passe, Horn, Stofan, \& Murray, 2004) and following exercise (Campagnolo et al., 2017; Wilmore, Morton, Gilbey, \& Wood, 1998). However, athletes may seek alternative fluid options following exercise, including beverages that contain alcohol (particularly at the recreational level) (O'Brien \& Lyons, 2000). Low-alcohol beer (LA-Beer) (i.e. $<1 \% \mathrm{ABV}$ ) is widely available, has been marketed as a recovery beverage (see https://int.erdinger.de/markenwelt/alkoholfrei/tea.html), and may make a valuable low-calorie contribution to fluid replacement following exercise (Maughan et al., 2016). Furthermore, in contrast to mid- and full-strength beer, LA-beer is unlikely to deliver a large absolute volume of alcohol and thus impair aspects of post-exercise recovery (e.g. rehydration (B Desbrow, Murray, \& Leveritt, 2013), or muscle protein resynthesis (Parr et al., 2014)). How access to different commercial beverages during the immediate post-exercise window influences acute voluntary fluid ingestion and subsequent nutrient intake by recreational athletes in a field setting remains unknown. 
50 Therefore, the aim of this investigation was to assess if providing different (but common)

51 beverages in a post-exercise recovery area influences voluntary fluid consumption, subsequent

52 dietary intake and next-morning hydration status. We hypothesised that (1): commercial sports

53 drinks would be consumed in greater quantities than either water or a LA-Beer, however, this

54 difference would not be sufficient to influence next-morning hydration status; and (2):

55 immediate access to a calorie-containing beverage (LA-Beer or sports drink) would result in

56 greater acute energy intakes post-exercise (compared to water) that would not be compensated

57 for by a reduction in food/fluid intake over the remainder of the day. 
The participant cohort consisted of consenting students who agreed to undertake a fluid and diet monitoring activity incorporated into an undergraduate sports nutrition course. Participants $(\mathrm{n}=132)$ completed two $10 \mathrm{~km}$ afternoon runs (repeated measures counterbalanced design) separated by one week. Immediately after the first run, participants were randomly assigned to separate recovery areas providing access to one beverage (based on personal preference). "Non-Beer Drinkers" ( $\mathrm{n}=78$ (38 male), mean \pm SD, age $=21.8 \pm 2.2 \mathrm{y}$, body mass $(\mathrm{BM})=71 \pm 13 \mathrm{~kg})$ received either Water or sports drink (SD) (Gatorade ${ }^{\circledR}$ (PepsiCo), lemon-lime flavour). "Beer Drinkers" ( $\mathrm{n}=54$ (41 male), age $=23.9 \pm 5.8 \mathrm{y}, \mathrm{BM}=76 \pm 13 \mathrm{~kg})$ received LA-Beer $\left(\right.$ Hahn Ultra ${ }^{\circledR}$, (LionCo), $0.9 \% \mathrm{ABV}$ ) or SD (Gatorade ${ }^{\circledR}$ (PepsiCo), orange flavour). Participants remained in the recovery area for 30-60 min and were given access to the alternate recovery beverage the following week. All fluid within the recovery area was consumed ad libitum and measured by trained observers (i.e. post-exercise fluid intake). Participants recorded all food and fluid consumed for the remainder of both trial days via food diary and photographs, which were subsequently analysed (energy, $\mathrm{CHO}$ and water) by a dietitian. Participants collected a next day waking urine sample to assess hydration status. The events commenced at the same time $\left(1400 \mathrm{hrs}\right.$ ), under similar environmental conditions (Trial $1=22.2^{\circ} \mathrm{C}, 63 \% \mathrm{RH}$ and Trial $2=$ $\left.25.8^{\circ} \mathrm{C}, 24 \% \mathrm{RH}\right)$ and were conducted on an athletics track (400m). Prior to data collection, all procedures were approved by the XXXX (removed for review) University Human Research Ethics Committee (HREC2017/351).

\section{Pre-trial Procedures}

On the morning of trials (consecutive Mondays), participants were encouraged to consume the same food. Once at the athletics track, participants self-categorized their pre-exercise dietary 
intake as "Nothing", "Fluids only", "Snack士Fluids", "Breakfast only", "Breakfast+Snack \pm Fluids”, "Breakfast+Lunch” or "Breakfast+Lunch + Snack \pm Fluids". Pre-

exercise intake was considered "matched" when it was reported within one ordinal category (e.g. Breakfast + Snack \pm Fluids $=$ Breakfast + Lunch $)$. Participants then provided a urine sample for the determination of USG (calibrated Pen Refractometers, ATAGO, USA) and urine color (8 point scale) (Armstrong et al., 1994). Immediately prior to commencing the run, participants self-reported their thirst and hunger (10 point scales), and recorded a nude BM.

\section{Experimental Procedures}

Participants were encouraged to complete the $10 \mathrm{~km}$ run at a sustainable pace able to be replicated in both trials. A combination of running and walking was permitted, however, participants were encouraged to run initially to induce fluid loss via sweating. To facilitate the calculation of fluid loss, participants were not allowed to drink throughout the task. Total distance and time were monitored by either GPS device (when available) or via lap counting and manual time keeping by one of the investigators.

On completion of the $10 \mathrm{~km}$ trial, participants repeated the thirst, hunger and nude BM measures before being allocated into a relevant treatment arm. The calorie-containing treatments supplied 103 vs $57 \mathrm{~kJ} \cdot 100 \mathrm{~mL}^{-1}$ (SD vs LA-Beer) of energy, 6 vs $1.8 \mathrm{~g} \cdot 100 \mathrm{~mL}^{-1}$ of CHO and 51 vs $3 \mathrm{mg} \cdot 100 \mathrm{~mL}^{-1}$ of sodium, respectively. The decision to use LA-Beer (rather than beer with higher $\% \mathrm{ABV}$ ) was partly due to ethical/safety concerns associated with managing the large participant cohort. All beverages were initially served cool $\left(\sim 10^{\circ} \mathrm{C}\right)$. Participants were required to stay in the recovery area for 30-60min and obtain fluid from bulk supplies (beer available on tap) using a standardized $(355 \mathrm{~mL})$ disposable cup. Participants were told to "drink as much as they wanted" and were able to refill (only empty) cups. Beverage liking and refreshingness were evaluated at the onset of drinking (on a scale of 1 to 10). All beverage 
volumes were recorded by weight (via digital scales), with participants instructed to return any unconsumed portion of their final drink for the determination of total beverage intake. Trained observers (student non-participants and investigators) monitored all drink volumes and compliance with drinking instructions. On leaving the recovery area, participants were provided with a urine specimen container for collection of a first morning sample the following day, used to determine $\mathrm{U}_{\mathrm{SG}}$ and Urine color.

\section{Dietary Analysis}

For both trial days, participants were instructed to record all food and fluid consumed via a food diary (including photographs), commencing from their departure of the recovery area until midnight. Participants were encouraged to include a self-selected fiducial marker (e.g. deidentified credit card) in each image to assist the investigator in the estimation of portion size. Completed food diaries/photos were analyzed for total energy $(\mathrm{kJ}), \mathrm{CHO}(\mathrm{g})$, and water $(\mathrm{L})$ by an experienced Accredited Practising Dietitian using Foodworks $9^{\circledR}$ (Xyris Software, Brisbane) dietary analysis software.

Planned comparisons employing paired-samples t-tests were used to assess differences in hydration and dietary outcome variables (Water vs SD and SD vs LA-Beer). Correlations between beverage "liking" and ad libitum consumption have been performed. Statistical significance was considered when $p<.05$. All data are Mean $\pm \mathrm{SD}$, where statistical differences existed, effect size (ES) was calculated as Cohen's $d$.

\section{Results}

127 Pre Trial: Despite advice to standardize pre-exercise food intake, 9 (7\%) participants (7 Water vs SD, 2 LA-Beer vs SD) had "unmatched" categories of food/fluid prior to trials. Therefore, 
all analysis was performed on both the entire dataset, and only those with "matched" preexercise food intake categories.

Whether the analysis was performed on all (Table 1) or only those with matched pre-exercise food intake categories did not influence any outcome variables (including those collected postexercise and the next-day). Furthermore, trial order analysis revealed no effect of trial sequence on body mass loss, run time, post-exercise subjective thirst/hunger ratings or drink volume consumed ( $p$ 's $>.05)$.

\section{INSERT Table 1 about here}

Water vs SD: Within the recovery area, both beverages were equally well received (liking $p=.420$ and refreshment $p=.089)$, and voluntarily consumed in similar quantities $(p=.157)$ (Table 1 and Figure 1). When provided access to SD, participants recorded a greater daily (recovery + rest of day) energy (av. $\Delta \sim 800 \mathrm{~kJ}, p=.002, \mathrm{ES}=.38$ ), $\mathrm{CHO}$ (av. $\Delta \sim 35 \mathrm{~g}, p<.001$, $\mathrm{ES}=.49)$ and fluid (av. $\Delta \sim 200 \mathrm{~mL}, p=.026, \mathrm{ES}=.26)$ intake.

\section{INSERT Figure 1 about here}

SD vs LA-Beer: SD was subjectively more enjoyable $(p<.001)$ and refreshing $(p<.001)$ than the LA-Beer, and voluntarily consumed in larger quantities (av. $\Delta \sim 200 \mathrm{~mL}, p=.004, \mathrm{ES}=.41$ ) (Table 1 and Figure 1). Participants recorded similar daily energy $(p=.591)$ and $\mathrm{CHO}(p=.833)$ intakes with both beverages. A small, but significant, reduction in total water intake (av. $\Delta$ $\sim 250 \mathrm{~mL}, p=.006$ ) remained at the end of the day following the LA-Beer trial.

Post Trial: Next morning $\mathrm{U}_{\mathrm{SG}}$ values were not different between Water vs SD (Water $=$ $1.020 \pm 0.009, \mathrm{SD}=1.025 \pm 0.037, p=.257)$. However, a difference was detected between SD vs LA-Beer $(\mathrm{SD}=1.021 \pm 0.009, \mathrm{LA}-\mathrm{Beer}=1.016 \pm 0.008, p=.002, \mathrm{ES}=.42)$. No differences were observed between any treatments for urine color. 
153 This study examined the impact of providing different beverages to recreational athletes following a self-paced $10 \mathrm{~km}$ run on acute ad libitum fluid consumption, subsequent dietary intake and next-morning hydration status. Results indicated that immediately following exercise, individuals voluntarily replaced $\sim 50-65 \%$ of the fluid lost via sweat, with the volume consumed associated with the palatability of the beverage. Water and commercial sports drink appeared equally well received, whereas, the low-alcohol beer was consumed in smaller volumes. When beverages differed in caloric density (i.e. SD vs Water), individuals did not compensate for the additional energy in the beverage by reducing subsequent post-exercise food/fluid intake. Beverage availability did not meaningfully influence next morning measures of hydration status.

In contrast to our hypothesis, the provision of a commercial SD did not result in significantly greater ad libitum fluid intakes compared to the Water trial during the immediate post-exercise period. Access to either of these beverages immediately following exercise resulted in similar intakes (i.e. $\Delta \sim 50 \mathrm{~mL}$ ). When provided throughout and after exercise, previous observations indicate that athletes voluntarily drink larger volumes (i.e. $\Delta$ 100-350 mL) of sweetened beverages compared to water (Passe, Horn, \& Murray, 2000; Passe et al., 2004; Wilmore et al., 1998). While statistically significant, these differences are likely to have a trivial impact on the fluid recovery of recreational athletes undertaking exercise in contexts similar to the current study (i.e. $\sim 2 \%$ body weight shift with considerable time between exercise bouts). Collectively, results suggest that consumption of SD or similarly sweetened beverages for rehydration purposes in this recreational setting is unwarranted.

The current study is the first to report on ad libitum intakes of LA-beer compared to SD following exercise. Results indicate that participants consumed $200 \mathrm{~mL}$ (on average) less LA- 
beer compared to SD in the immediate post-exercise period. The same relationship was evident when the analysis was conducted exclusively on the male participants (results not provided). Despite participant's being informed of the "low" alcohol content of the beer, it is not possible to determine if concerns with the alcohol per se influenced consumption (i.e. either by avoiding less "socially desirable" LA-beer or trepidations regarding alcohol intake from sceptical participants). That said, the reduced volume can, in part, be explained by lower palatability (average rating $\sim 1.5$ lower out of 10 ) reported during the LA-Beer trials (see supplementary figure). Furthermore, carbonation has previously been associated with reduced voluntary fluid consumption following exercise (Passe, Horn, \& Murray, 1997). Interestingly, the fluid deficit observed on LA-Beer trials further increased to $\sim 350 \mathrm{~mL}$ by the end of the day. This suggests that maximising fluid intakes during the immediate post-exercise period (i.e. providing access to palatable fluids) assists in optimising fluid intakes over the remainder of the day.

While the results of our previous investigation indicated that a recovery intervention did not influence total energy or macronutrient intake, this result was in the context of numerous fluid/food items (varying in calorie density) being available. The current design considered the impact of providing one beverage within a recovery area (as might be the case under funding constraints). In support of our hypothesis, the exclusive provision of a calorie-containing beverage (either SD or LA-Beer) appeared to influence energy intake values recorded at the end of trial days. In fact, the energy/CHO surplus created by the provision of SD vs Water (mean $\sim 850 \mathrm{~kJ} / \sim 45 \mathrm{~g}$ ) was almost completely preserved until the end of our recording period. This finding is consistent with recent laboratory work (using trained participants) indicating that the acute energy provision provided by caloric beverages is not typically offset by a subsequent reduction in food/fluid intake over the remainder of a day (Campagnolo et al., 2017; McCartney et al., 2018). 

benefits of healthy eating with regular physical activity (i.e. a "teachable moment" for health advocacy (Lawson \& Flocke, 2009)), and (3): if the intention is to increase energy intake postexercise (e.g. multi-day (competitive or charity-type) events), the provision of caloriecontaining fluids (including a low-alcohol beer) may be warranted.

\section{Conclusion}

The exclusive provision of calorie-containing drinks (compared to water) following exercise influences subsequent dietary intake, with minimal impact on next day hydration in recreational runners.

\section{References}

Armstrong, L. E., Maresh, C. M., Castellani, J. W., Bergeron, M. F., Kenefick, R. W., LaGasse, K. E., \& Riebe, D. (1994). Urinary indices of hydration status. Int J Sport Nutr, 4(3), 265-279.

Burke, L. M., van Loon, L. J. C., \& Hawley, J. A. (2017). Postexercise muscle glycogen resynthesis in humans. J Appl Physiol (1985), 122(5), 1055-1067. doi:10.1152/japplphysiol.00860.2016

Campagnolo, N., ludakhina, E., Irwin, C., Schubert, M., Cox, G. R., Leveritt, M., \& Desbrow, B. (2017). Fluid, energy and nutrient recovery via ad libitum intake of different fluids and food. Physiol Behav, 171, 228-235. doi:10.1016/j.physbeh.2017.01.009

Desbrow, B., Barnes, K., Young, C., Cox, G. R., \& Irwin, C. (2017). A Nutrition Recovery Station Following Recreational Exercise Improves Fruit Consumption but Does Not Influence Fluid Recovery. Int J Sport Nutr Exerc Metab, 27(6), 487-490. doi:10.1123/ijsnem.2017-0294

Desbrow, B., Murray, D., \& Leveritt, M. (2013). Beer as a Sports Drink? Manipulating Beer's Ingredients to Replace Lost Fluid. International Journal of Sport Nutrition and Exercise Metabolism, 23(6), 593-600.

Lawson, P. J., \& Flocke, S. A. (2009). Teachable moments for health behavior change: a concept analysis. Patient Educ Couns, 76(1), 25-30. doi:10.1016/j.pec.2008.11.002

Maughan, R. J., Watson, P., Cordery, P. A., Walsh, N. P., Oliver, S. J., Dolci, A., . . Galloway, S. D. (2016). A randomized trial to assess the potential of different beverages to affect hydration status: development of a beverage hydration index. Am J Clin Nutr, 103(3), 717-723. doi:10.3945/ajen.115.114769

McCartney, D., Irwin, C., Cox, G. R., \& Desbrow, B. (2018). Fluid, Energy and Nutrient Recovery via Ad Libitum Intake of Different Commercial Beverages and Food in Female Athletes. Appl Physiol Nutr Metab. doi:10.1139/apnm-2018-0176

O'Brien, C. P., \& Lyons, F. (2000). Alcohol and the athlete. Sports Med, 29(5), 295-300. doi:10.2165/00007256-200029050-00001 
Parr, E. B., Camera, D. M., Areta, J. L., Burke, L. M., Phillips, S. M., Hawley, J. A., \& Coffey, V. G. (2014). Alcohol ingestion impairs maximal post-exercise rates of myofibrillar protein synthesis following a single bout of concurrent training. PLoS One, 9(2), e88384. doi:10.1371/journal.pone.0088384

Passe, D., Horn, M., \& Murray, R. (1997). The effects of beverage carbonation on sensory responses and voluntary fluid intake following exercise. International Journal of Sport Nutrition, 7, 286297.

Passe, D., Horn, M., \& Murray, R. (2000). Impact of beverage acceptability on fluid intake during exercise. Appetite, 35, 219-229.

Passe, D., Horn, M., Stofan, J., \& Murray, R. (2004). Palatability and Voluntary Intake of Sports Beverages, Diluted Orange Juice, and Water During Exercise. International Journal of Sport Nutrition and Exercise Metabolism, 14, 266-278.

Wilmore, J. H., Morton, A. R., Gilbey, H. J., \& Wood, R. J. (1998). Role of taste preference on fluid intake during and after 90 min of running at $60 \%$ of VO2max in the heat. Med Sci Sports Exerc, 30(4), 587-595. 